\title{
Pré-processamento de sinais Eletro-oculográficos (EOG)
}

\author{
Pre-processing of Electrooculography (EOG) \\ Preprocesamiento de señales Electrooculográficas (EOG)
}

Recebido: 23/03/2021 | Revisado: 29/03/2021 | Aceito: 30/03/2021 | Publicado: 10/04/2021

\author{
Gilberto de Melo Junior \\ ORCID: https://orcid.org/0000-0002-2317-7779 \\ Instituto Federal do Pará, Brasil \\ E-mail: gilberto.melo@ifpa.edu.br \\ Sílvio Leão Vieira \\ ORCID: https://orcid.org/0000-0003-4523-0769 \\ Universidade Federal de Goiás, Brasil \\ E-mail: slvieira@ufg.br \\ Geovanne Pereira Furriel \\ ORCID: https://orcid.org/0000-0002-8510-1024 \\ Instituto Federal Goiano, Brasil \\ E-mail: geovanne.furriel@ifgoiano.edu.br \\ Renato Milhomem de Oliveira Filho \\ ORCID: https://orcid.org/0000-0003-3351-1176 \\ Instituto Federal de Goiás, Brasil \\ E-mail: rmoliveira17@gmail.com \\ Brunna Carolinne Rocha Silva \\ ORCID: https://orcid.org/0000-0003-1654-5980 \\ Instituto Federal de Goiás, Brasil \\ E-mail: brunnac.rocha@gmail.com \\ Jane Sousa Naves \\ ORCID: https://orcid.org/0000-0003-4463-4462 \\ Universidade Federal de Goiás, Brasil \\ E-mail: janenaves97@gmail.com
}

\begin{abstract}
Resumo
Este trabalho visa estudar sinais eletrooculográficos (EOG) provenientes da aquisição, incluindo janelamento, e aplicação de filtros digitais para processamento e análise. EOG é um sinal que mede e registra o potencial de repouso da retina do olho. Aqui, desenvolvemos um algoritmo para detectar sinais de movimento do globo ocular relativos a eletrodos fixados na face. Os sinais bioelétricos foram coletados de 40 voluntários adultos saudáveis. A metodologia aplicada segue as normas e diretrizes internacionais para aquisição de dados de sinais de ECG e com a anuência do Comitê de Ética em Pesquisa institucional. Dois filtros digitais Savitzky-Golay (SG) e Median Filter (FM), foram comparados. Como resultado, foram desenvolvidos e registrados softwares para janelamento de sinais e comparação entre filtros digitais, sendo o Savitzky-Golay o mais adequado para uso com EOG.
\end{abstract}

Palavras-chave: Pré-processamento; Sinais eletro-oculográficos; Filtro Savitzky-Golay; Filtro mediana.

\begin{abstract}
This work aims to study electrooculographic signals (EOG) from the acquisition, including windowing, and application of digital filters for processing and analysis. EOG is a signal that measures and records the resting potential of the eye retina. Herein, we develop an algorithm to detect eyeball movement signals relative to electrodes fix to face. The bioelectric signals were collected from 40 healthy adult volunteers. The applied methodology follows the international standards and guidelines for data acquisition of ECG signals and with the agreement of the institutional Research Ethics Committee. Two digital filters Savitzky-Golay (SG) and Median Filter (FM), were compared. As a result, were development and registration of software for windowing signals and the comparison between digital filters, with Savitzky-Golay being the most suitable for using with EOG.
\end{abstract}

Keywords: Pre-processing; Electrooculographic signals; Savitzky-Golay filter; Median filter.

\section{Resumen}

Este trabajo tiene como objetivo el estudio de las señales electrooculográficas (EOG) de la adquisición, incluido el sistema de ventanas, y la aplicación de filtros digitales para su procesamiento y análisis. EOG es una señal que mide y registra el potencial de reposo de la retina del ojo. Aquí, hemos desarrollado un algoritmo para detectar señales de movimiento del globo ocular en relación con los electrodos fijados en la cara. Se recogieron señales bioeléctricas de 40 voluntarios adultos sanos. La metodología aplicada sigue las normas y directrices internacionales para la adquisición de datos de señales de ECG y con el consentimiento del Comité de Ética de Investigación Institucional. Se 
compararon dos filtros digitales Savitzky-Golay (SG) y Median Filter (FM). Como resultado, se desarrolló y registró un software para ventanas de señal y comparación entre filtros digitales, siendo Savitzky-Golay el más adecuado para su uso con EOG.

Palabras clave: Preprocesamiento; Señales electrooculográficas; Filtro Savitzky-Golay; Filtro mediano.

\section{Introdução}

A utilização de tecnologias de aquisição e armazenamento de dados tem-se desenvolvido nas mais diversas áreas do conhecimento. Diversos métodos têm sido propostos para o processamento de dados com o objetivo de extrair conhecimento das informações contida nos dados (Lee, 2005). No campo dos sinais biomédicos, aqueles de natureza bioelétrica são os mais contaminados por ruído. Assim, os fenômenos de despolarização de células, tais como as cerebrais, cardíacas, musculares e ocular são captadas na superfície da pele e possuindo uma baixa relação sinal-ruído (SNR). No caso ocular o sinal bioelétrico gerado pelo movimento dos olhos é conhecido por eletro-oculografia (EOG).

De acordo com Crescentini et al. (2014), o ruído representa uma grande preocupação no processamento e extração de informações (Chaudhuri et al., 2012). Na literatura da área são encontrados diversos trabalho onde relatam que a principal interferência nos sinais de EOG é o ruído do sensor, o ruído da linha de energia, interferência dos movimentos da cabeça e da fala.

No trabalho de Kim et al. (2007) foi proposto um algoritmo que discrimina vários movimentos oculares dos sinais EOG. O foco não são apenas movimentos sacádicos ou piscadas, mas também piscadas duplas / triplas e piscadas esquerda / direita. Além disso, no tratamento dos sinais é sugerido um novo método que remove os ruídos do EOG, para aumentar a robustez da discriminação. Com a aplicação deste novo método, foi possível a obtenção dos movimentos oculares e foram usados para uma Interação/Interface Humano-Computador (IHC) entre uma pessoa e um robô móvel. Como citado, no trabalho de Kim et al. (2007), remoção de ruídos e tratamento dos sinais EOG é de grande importância.

Mala e Latha (2016) investigam a redução de ruído dedicada a sinais EOG como um pré-processamento de dados para posteriormente ser reconhecido como atividade. Este trabalho se concentra na redução de ruído com base na Transformada de Wavelet Discreta (DWT) para remover o ruído e melhorar a identificação dos recursos do sinal EOG.

Também neste sentindo, Choudhury et al. (2005) descrevem um projeto de desenvolvimento de um novo sistema de aquisição de sinais EOG que otimiza os sinais em relação aos ruídos característico deste tipo de sinal, tornando-o adequado para análises teóricas e aplicações industriais.

Dessa forma, o processo de filtragem do EOG é desafiador devido à estrutura inerente não supervisionada do ruído (Vogt, 2015). Bhandari et al. (2006) descrevem que é importante filtrar os sinais biomédicos para obter estimativa precisa dos parâmetros, aumentar a precisão do diagnóstico e prognóstico médico, e fornecer melhor interpretação das informações dos sinais.

Nesse trabalho é apresentado ferramentas de pré-processamento de sinais EOG, incluindo janelamento e filtragem sinais EOG com o objetivo de realçar a relação sinal-ruído. De forma resumida, o artigo está estruturado na seção de Materiais e Métodos, onde são apresentadas subseções 2.1 Aquisição de sinais EOG; subseção 2.2, Janelamento de sinais EOG; subseção 2.3, Filtragem de sinais EOG. Na seção Resultados são apresentados os dados obtidos nos experimentos. Finalmente, na seção Conclusões são discutidos os resultados e possíveis trabalhos futuros.

\section{Materiais e Métodos}

Nesta seção é apresentado as etapas de pré-processamento aplicadas aos sinais EOG. Primeiramente foi apresentado como realizou a aquisição dos sinais EOG. Após a aquisição houve o janelamento dos sinais EOG. Com o janelamento feito, houve então as operações com os atributos dos sinais. Por último apresentou a aplicação de filtros digitais. 
Este artigo, de acordo com Pereira et al. (2018) apresenta uma pesquisa qualitativa, no sentindo de análise numérica dos dados obtidos.

\subsection{Aquisição de sinais EOG}

Na aquisição dos sinais foi utilizado o Miotool 200/400 USB da Miotec Equipamentos Biomédicos Ltda, RS, Brasil. Ele é um equipamento que permite a aquisição de sinais de eletromiografia de superfície. $\mathrm{O}$ dispositivo funciona em conjunto com o software Miograph USB. De acordo com o manual do equipamento, o sistema atende todos os registros da norma de segurança para equipamentos eletromédicos NBR IEC 601- 1/1994 e EMENDA (1997), NBR IEC 60601-1-2: 2006 e Norma Particular NBR IEC 60601-2-40/1998 (Miotec Equipamentos Biomédicos, 2010).

O dispositivo apresenta as seguintes especificações (Miotec Equipamentos Biomédicos, 2010):

- 14 bits de resolução;

- Taxa de aquisição por canal de 2000 amostras por segundo;

- Baixo nível de ruído;

- Modo de rejeição comum de $110 \mathrm{~dB}$;

- Isolamento de segurança 3000 Vrms.

Para conexão entre os elétrodos e o Miotool 200/400 USB, foi utilizado o Sensor Diferencial de Superfície (SDS500) com conexão por garras. Esse tipo de conexão é apropriado para elétrodos que são fixados em lugares não planos e de difícil fixação, como por exemplo, o rosto. Assim, o SDS500 tipo garra permite ajustes na colocação dos elétrodos facilitando a aquisição dos sinais (Miotec Equipamentos Biomédicos, 2010).

O software Miograph USB foi previamente configurado para a aquisição dos sinais EOG. Para o tipo de aquisição, foi escolhida a opção sEMG S, referente aos sinais EMG de superfície da pele. Foram utilizados dois canais de aquisição do equipamento, sendo que cada canal é interligado por duas entradas de eletrodos. Além dos eletrodos dos dois canais, também houve o eletrodo de referência conectado a uma saída específica do equipamento. As principais configurações, para ambos os canais, são:

- Filtro Passa Alta de 0,1 Hz de quarta ordem;

- Filtro Passa Baixa de $40 \mathrm{~Hz}$ de quarta ordem;

- Filtro Notch $60 \mathrm{~Hz}$ de quarta ordem;

- $\quad$ CVM configurado para o máximo $900 \mu \mathrm{V}$;

Sendo um software proprietário, ele possui duas maneiras de trabalhar com a apresentação dos resultados: gerando o laudo em formato .pdf ou arquivando os sinais em arquivos. EXMS.

\subsection{Janelamento de sinais EOG}

Os sinais das amostras foram adquiridos em formato. EXMS, um formato não compatível com o ambiente de processamento empregado. Para manusear os sinais adquiridos com maior eficiência, foi realizada a conversão dos sinais. EXMS para. MAT.

Dessa forma, os sinais passaram a ser representados em vetores numéricos. Cada vetor, que corresponde a uma amostra de sinal EOG obtido de um voluntário, possui 120.000 números de pontos amostrados. Uma amostra de sinal por sua vez possui uma duração de 60 segundos, sendo que cada porção de segundo é composto por 2000 números de pontos amostrados. O método utilizado para detecção do movimento ocular, extrai a soma das médias de amplitude de cada segundo de amostra analisado.

Por exemplo, caso a média de amplitude naquele segundo for maior do que a média de amplitude do sinal global, o 
algoritmo identifica como um sinal de movimento ocular. Alguns dos movimentos oculares referenciados nas sequências geram alterações na amplitude do sinal maiores do que um segundo. Assim, para adquirir o sinal completo, foi agrupado o segundo em que detectou essa variação mais dois segundos à frente, tornando pequenos sinais de 3 segundos (6000 pontos amostrados). Esse procedimento foi realizado para os sinais de ambos os canais de aquisição. No Algoritmo 1 é apresentado o passo-a-passo do código implementado.

Algoritmo 1. Separação dos sinais de movimentos oculares.

Entrada: Vetor da amostra de sinal EOG de um voluntário.

Saída: Vetores de amostras de sinais que apresentam movimentos oculares.

Início

Passo 1: Dividir o sinal coletado em amostras de segundos (uma amostra contém $60 \mathrm{~s}$ segundos e cada segundo 2000 pontos de amostra);

Passo 2: Efetuar a soma dos sinais em cada amostra de segundo;

Passo 3: Encontrar a média dos sinais em cada amostra de segundo;

Passo 4: Montar uma célula com cada variação do sinal encontrada, utilizando a média de todo o sinal (na amostra de segundo em que notou a variação mais 2 amostras de segundo para frente);

Passo 5: Transformar a célula em matriz com cada sinal encontrado;

Passo 6: Separar os sinais da matriz em vetores;

Passo 7: Plotar os sinais de movimentos oculares retirados do sinal inicial;

Fim

\subsection{Filtragem de sinais EOG}

No processamento de sinal, a função de um filtro é remover partes indesejadas do sinal, como ruído aleatório, ou extrair partes úteis do sinal, como os componentes localizados dentro de uma determinada faixa de frequência. Um filtro digital usa um processador digital para executar cálculos numéricos nos valores amostrados do sinal. O processador pode ser um computador de uso geral ou um chip Digital Signal Processor (DSP) especializado.

Os filtros digitais são vantajosos em relação aos analógicos pois podem ser facilmente programados, desenhados e são mais versáteis (Lathi, 2006). Neste trabalho foram utilizados dois filtros digitais: Savitzky-Golay (SG) e Filtro da Mediana (FM).

Esses filtros já foram utilizados na literatura aplicados aos biossinais. Awal et al. (2011) apresentaram o filtro Savitzky-Golay aplicado a sinais Eletrocardiográficos. Já Agarwal et al. (2016) descrevem a aplicação do SG ao préprocessamento em tempo real de um sistema médico inteligente para diagnósticos. Já o filtro FM pode ser observado no trabalho de Bulling et al. (2010), onde descrevem uma análise dos movimentos dos olhos para atividades de reconhecimento usando sinais EOG.

O filtro digital de Savitzky-Golay foi criado em 1964 como um algoritmo de filtragem e suavização de dados, incluindo as derivadas do sinal, e pode ser pensado como uma generalização de um filtro de média móvel. Esse filtro foi introduzido pela primeira vez por Savitzky e Golay na química analítica como uma solução para suavizar os dados ruidosos obtidos do analisador de espectro químico (Sadeghi \& Behnia, 2018).

Os coeficientes desse filtro são provenientes da realização de um ajuste linear não-ponderado de mínimos quadrados, utilizando um polinômio de determinada ordem no ponto central da janela (Zhao et al., 2014). O filtro pode ser descrito pela Equação (1) (Nishida, 2017): 
$s_{i}=\frac{1}{2 m+1} \sum_{j=-m}^{m} w_{j} s_{i+j}$

em que $\mathrm{s}_{i}$ é o valor suavizado no filtro; $\mathrm{m}$ é o ponto médio da janela de amostras; e $\mathrm{w}_{i}$ o valor dos coeficientes do filtro.

O filtro da Mediana (FM) é um filtro de janela de classe não linear, que remove facilmente ruídos destrutivos e preserva as bordas do sinal. A ideia básica por trás do filtro é que qualquer elemento do sinal (que pode ser também uma imagem) observe sua vizinhança e escolha o elemento mais semelhante aos outros (Boateng et al., 2012).

O filtro de mediana em suas propriedades se assemelha ao filtro médio, entretanto mais eficiente no tratamento de ruído e preservação de bordas. Bulling et al., (2010) observaram que o filtro mediano aplicado em sinais EOG apresentou as seguintes características: preservar a margem íngreme dos movimentos oculares sacádicos, reteve as amplitudes do sinal de EOG e não introduziu alterações artificiais no sinal.

É importante escolher um tamanho de janela que seja pequeno o suficiente para reter pulsos curtos de sinal, particularmente aqueles causados pela piscada. Um filtro mediano remove pulsos de largura menor que cerca da metade do tamanho da janela (Bulling et al., 2010).

Ambos filtros foram escolhidos por serem aplicados a sinais biomédicos, como descrito na literatura da área (Dasgupta et al., 2017), (Awal et al., 2011), (Bulling et al., 2010). Aqui, a configuração dos filtros baseou-se em estudos previamente publicados. Por exemplo, Dasgupta et al. (2017) descrevem o desenvolvimento de um framework de filtragem de sinais EOG. Empiricamente, este trabalho utiliza o tamanho de janela em número de pontos do filtro de mediana igual a 9.

Sadeghi e Behnia (2018), em seus estudos de otimização de filtros Savitsky-Golay, afirmaram que as ordens iguais ou maiores a 2 não apresentam diferenças expressivas no uso deste filtro e que o tamanho de janela pode ser variado, dependendo do sinal. Foram utilizados neste trabalho a ordem de 15 e tamanho de janela igual a 331, seguindo especificações encontradas em (Sadeghi \& Behnia, 2018).

\section{Resultados}

Antes da aquisição dos sinais EOG, o projeto foi submetido e aprovado pelo Comitê de Ética em Pesquisa (CEP) da Universidade Federal de Goiás (UFG) sob o Certificado de Apresentação para Apreciação Ética (CAAE), número 15226219.2.0000.5083 e sob o Número de Parecer 3.521.755.

Os dados foram coletados utilizando o dispositivo Miotool 200/400 USB em conjunto com o software Miograph USB. Em seguida os dados foram exportados em formato. EXMS, formato de arquivo padrão empregado pelo dispositivo. Para que os dados brutos pudessem ser analisados e processados, foram necessários alguns passos:

a) extraiu-se o arquivo. EXMS utilizando o software livre 7zip;

b) o arquivo foi convertido para o formato. edf. Para abri-lo, utilizou-se o software livre EDFbrowser;

c) no menu do EDFbrowser, na aba Tools, converteu-se o arquivo de. edf/bdf para ASCII(CSV);

d) estando neste formato, foi possível importar para um ambiente convencional de processamento de dados, tal como Scilab ou MatLab, onde os sinais passaram a ser representados por vetores, facilitando os próximos passos do préprocessamento.

As Figuras 1 e 2 ilustram exemplos de amostras de sinais EOG, respectivamente do canal 1 e do canal 2, obtidos durante a pesquisa. 
Figura 1. Exemplo de amostra de um sinal EOG coletado no canal 1.

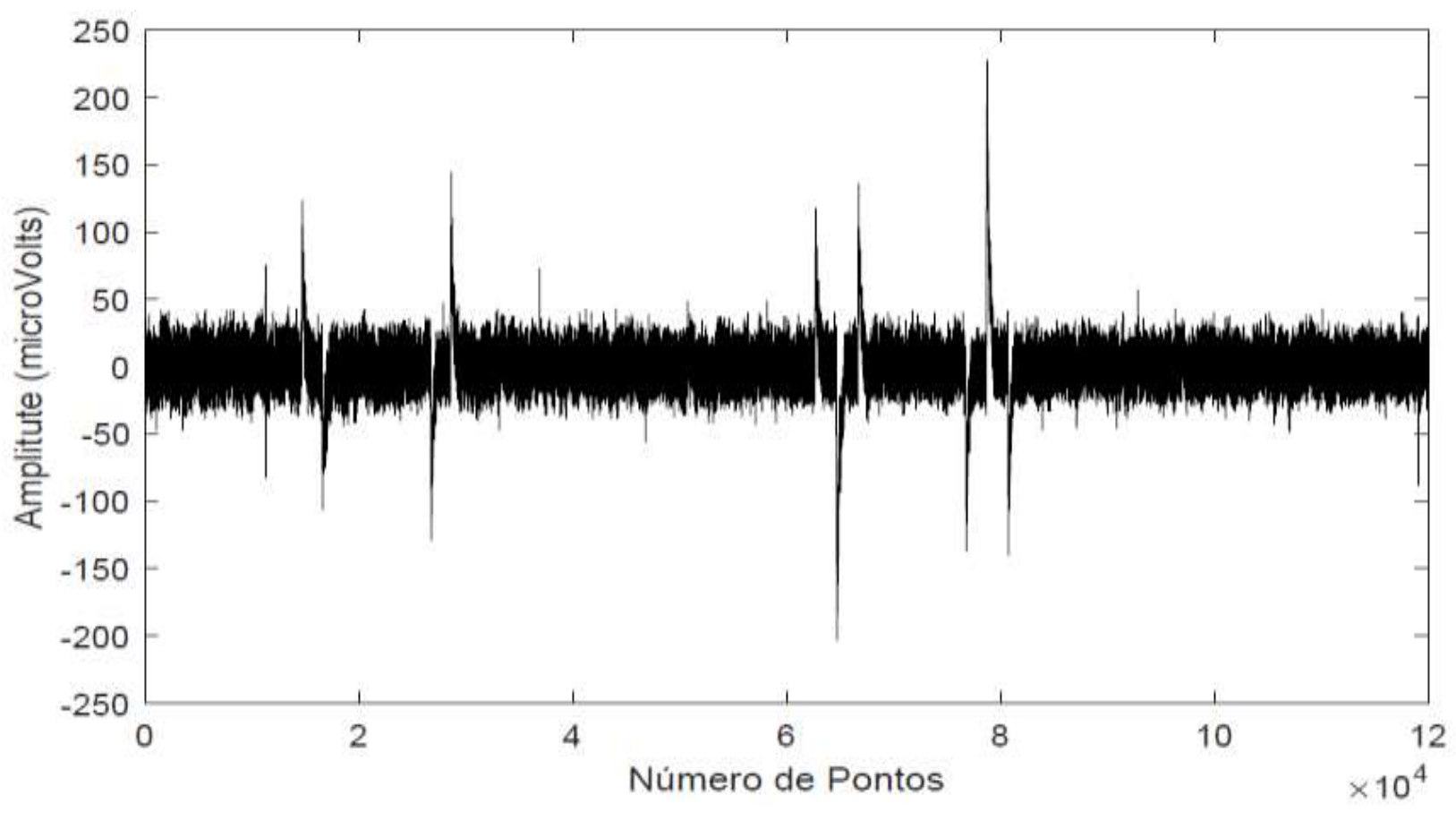

Fonte. Autores.

Figura 2. Exemplo de amostra de um sinal EOG coletado no canal 2.

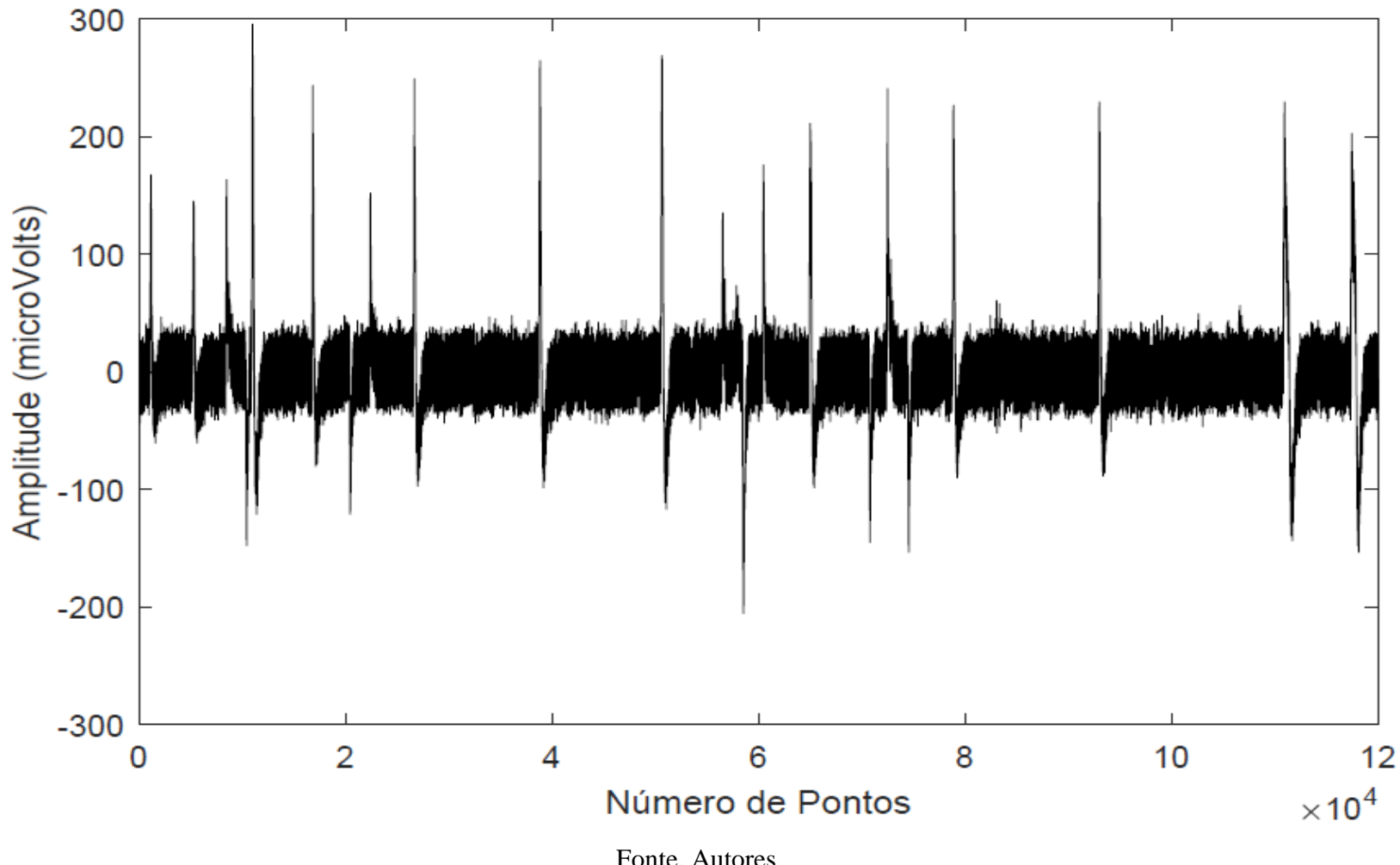

Com a aplicação do algoritmo de janelamento descrito na Subseção 2.2, pode-se extrair os movimentos oculares pretendidos na aquisição dos sinais EOG. Figura 3 apresenta alguns desses movimentos oculares janelados da amostra de sinal EOG através do algoritmo desenvolvido. 
Figura 3. Exemplos de sinais de movimentos oculares para ambos canais 1 e 2, após a aplicação do algoritmo de janelamento de sinais.
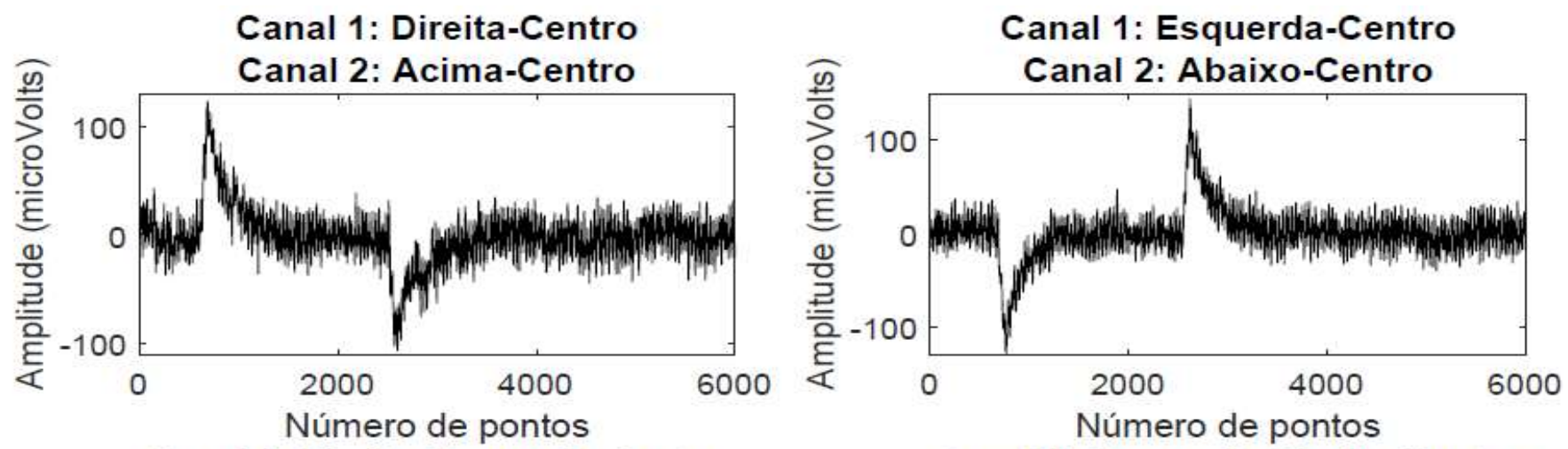

Canal 1: Direita-Esquerda-Centro
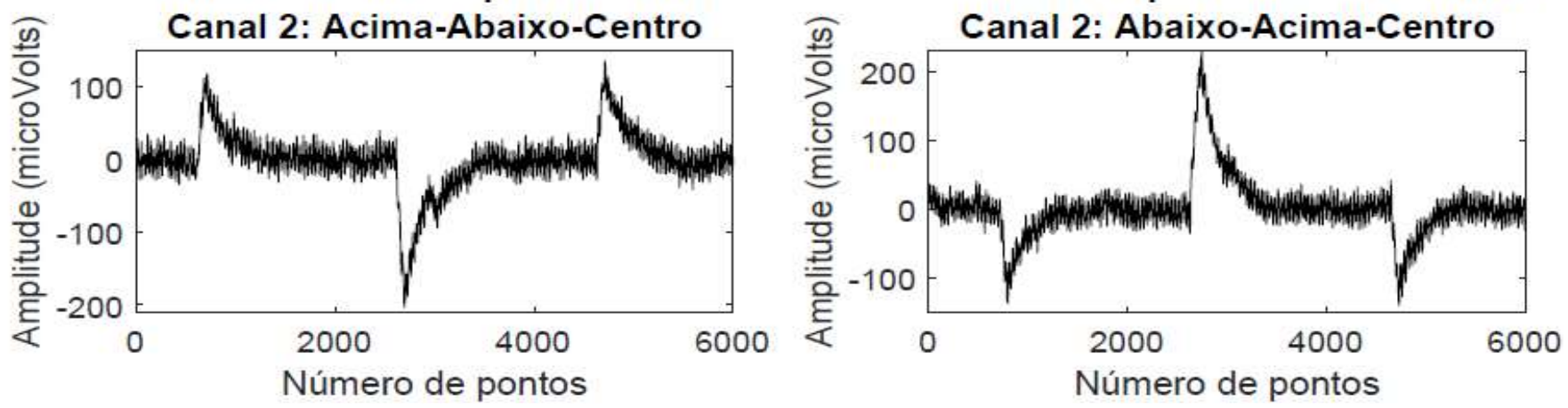

Fonte. Autores.

Com o janelamento dos movimentos oculares dos sinais EOG desenvolvido, o passo seguinte foi a aplicação dos filtros digitais nas amostras de sinais. A Figura 4 ilustra a percepção da amostra de sinal EOG coletada do voluntário e o sinal após aplicação dos filtros digitais Savitzky-Golay (SG) e Filtro da Mediana (FM). A Figura 5 ilustra uma visualização do sinal ampliada para que se possa notar com maior nitidez a diferença entre os filtros. 
Figura 4. Comparação do sinal bruto (sem filtros digitais), com o filtro FM e com o filtro SG.

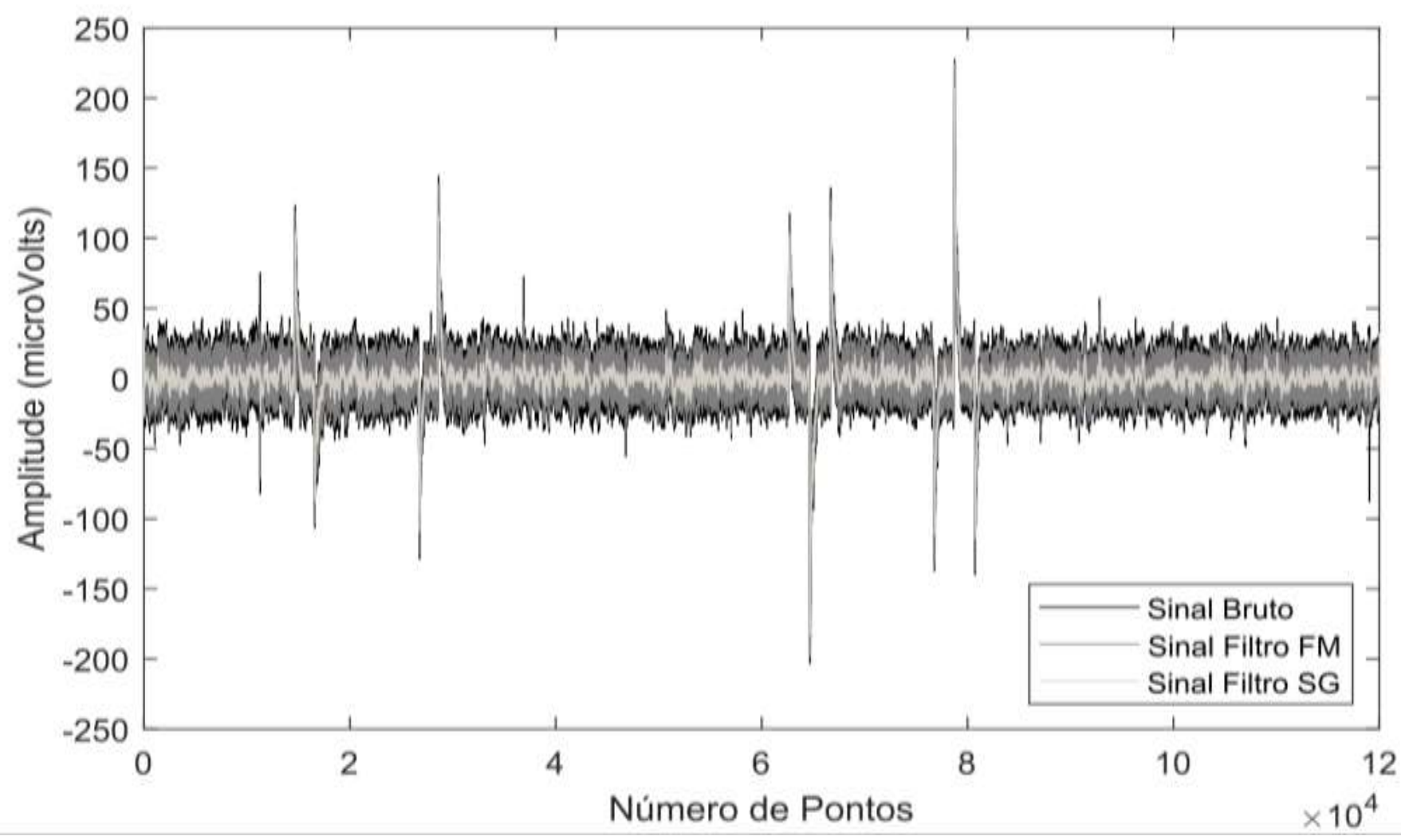

Fonte. Autores.

Figura 5. Comparação de sinais com filtro com visualização ampliada.

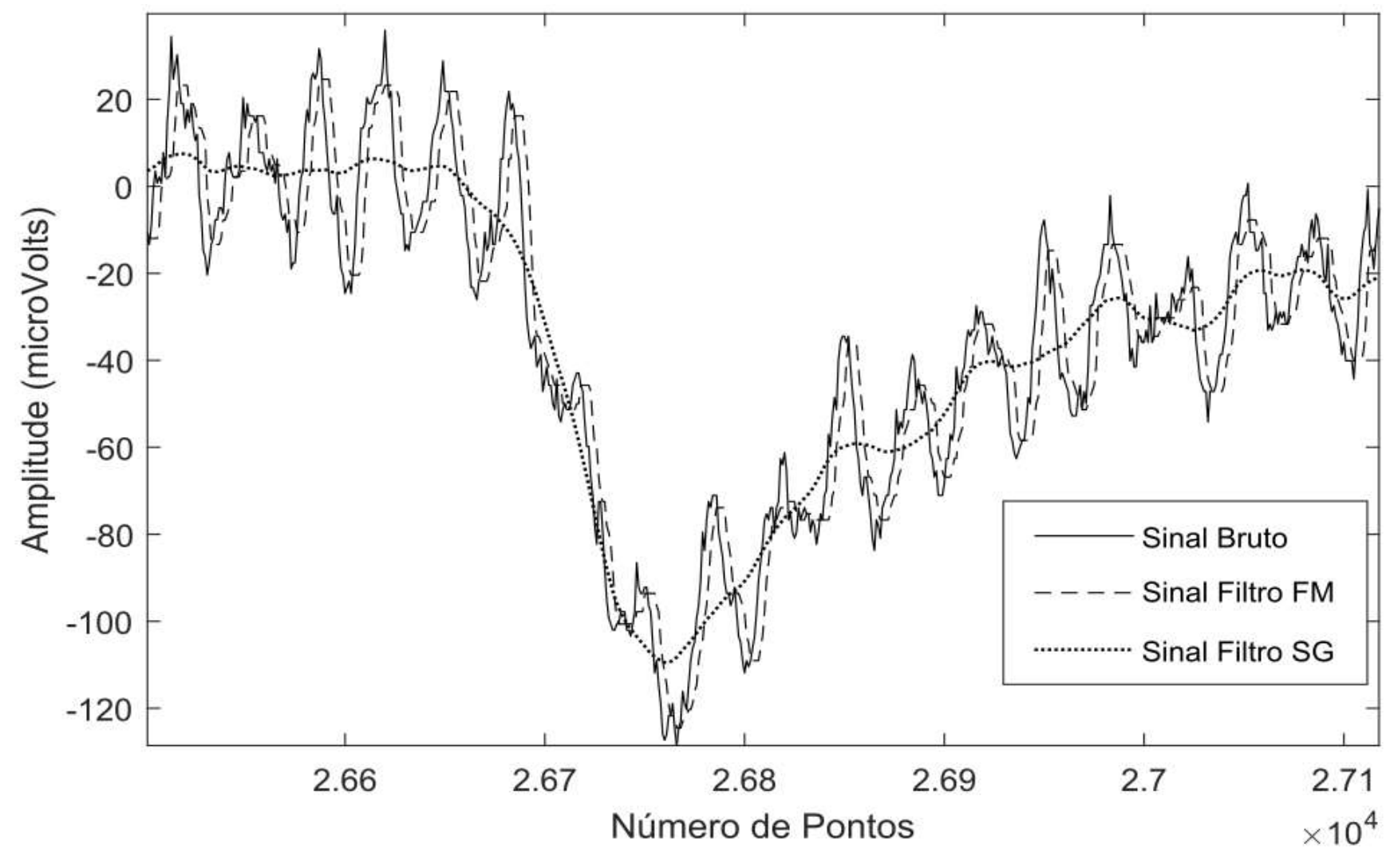

Fonte. Autores. 
A aquisição dos sinais EOG foi realizada em duas fases. A primeira com o voluntário exposto a luz (100 lux) e a segunda com ausência de luz. De acordo com o Padrão Clínico de Eletro-oculográfia escrito pela Internacional Society for Clinical Electrophysiology of Vision (ISCEV) (Constable et al., 2017), entre 7 e 12 minutos nessa etapa há um registro de aumento de potencial bioelétrico no olho, atingido assim o que é chamado de Pico Luminoso (PL). Na segunda fase, entre 10 e 15 minutos de ausência de luz, há uma queda do potencial, atingindo assim o que pode ser chamado de Vale Escuro (VE).

Assim de acordo com a norma apresentada, foi realizado o teste de aquisição entre 10 e 12 minutos para cada fase. Para a validação dos sinais coletados, o padrão ainda recorre a relação entre o máximo valor de PL e o mínimo valor de VE, razão PL/VE. Até alguns anos essa razão era conhecida como Índice de Arden (Zago, 2010) e, tipicamente, deve estar entre 1,7 e 4,3. De acordo com Constable et al. (2017), cada pesquisa deve estabelecer seu próprio conjunto de valores normativos, porém é sugerido que o valor da razão PL/VE fique entre os valores sugeridos ou próximos deles.

Para comparação dos filtros aplicados foi utilizado a razão PL/VE, extraindo a mediana das amostras coletadas. O Quadro 1 apresenta os valores das razões PL/VE encontrados nos sinais brutos (sem filtros digitais), com filtro SG e com filtro FM. Os valores referentes são de apenas 40 amostras de sinais, sendo uma de cada indivíduo.

Quadro 1. Razão PL/VE dos sinais EOG coletados.

\begin{tabular}{|c|c|c|c|}
\hline Sinais & Canal 1 & Canal 2 & Ambos os canais \\
\hline Sem filtro & 1,57 & 1,40 & 1,48 \\
\hline Filtro SG & 1,45 & 1,46 & 1,46 \\
\hline Filtro MF & 1,39 & 1,49 & 1,43 \\
\hline
\end{tabular}

Fonte. Autores.

Os valores da razão PL/VE ficaram bem próximos ao sugerido, com o filtro SG sendo os valores mais próximos do que o filtro MF. Sugerindo assim a melhor aceitação dos sinais EOG para o filtro SG.

\section{Conclusão}

Este trabalho propôs um estudo de sinais EOG, desde a aquisição dos sinais, ao pré-processamento com a aplicação de filtros digitais e janelamento de sinais para a aquisição dos movimentos oculares. Foi possível o desenvolvimento de um algoritmo para janelamento de sinais EOG, sendo bastante funcional a sua aplicação. Foi realizada uma análise comparativa entre dois filtros digitais Savitzky-Golay (SG) e Filtro da Mediana (FM). Como resultado preliminar foi observado uma melhor relação sinal-ruído dos sinais de EOG quando aplicado o filtro SG.

O trabalho gerou o registro de um software no Instituto Nacional de Propriedade Intelectual (INPI), registrado em 28/01/2020 intitulado: BSPS - Software de processamento de sinais biomédicos. Patente: Programa de computador. Número de registro: BR512020000172-6, ano de 2020.

Para trabalhos futuros, os autores sugerem a aplicação de algoritmos de Machine Learning ou Deep Learning para classificação dos sinais de movimentos oculares separados pelo algoritmo de janelamento de sinais.

\section{Agradecimentos}

Os autores agradecem o suporte da Universidade Federal de Goiás (UFG), Coordenação de Aperfeiçoamento de Pessoal de Nível Superior (CAPES), Conselho Nacional de Desenvolvimento Científico e Tecnológico (CNPq) e a Fundação de Amparo à Pesquisa do Estado de Goiás (FAPEG). 
Research, Society and Development, v. 10, n. 4, e28410414221, 2021

(CC BY 4.0) | ISSN 2525-3409 | DOI: http://dx.doi.org/10.33448/rsd-v10i4.14221

\section{Referências}

Agarwal, S., Rani, A., Singh, V., \& Mittal, A. P. (2016). Performance evaluation and implementation of FPGA based SGSF in smart diagnostic applications. Journal of medical systems, 40(3), 63.

Awal, M. A., Mostafa, S. S., \& Ahmad, M. (2011). Performance analysis of Savitzky-Golay smoothing filter using ECG signal. International Journal of Computer and Information Technology, 1(02), 24.

Bhandari, A., Khare, V., Trikha, M., \& Anand, S. (2006, September). Wavelet based novel technique for signal conditioning of electro-oculogram signals. In 2006 Annual IEEE India Conference (pp. 1-6). IEEE.

Boateng, K. O., Asubam, B. W., \& Laar, D. S. (2012). Improving the effectiveness of the median filter.

Bulling, A., Ward, J. A., Gellersen, H., \& Tröster, G. (2010). Eye movement analysis for activity recognition using electrooculography. IEEE transactions on pattern analysis and machine intelligence, 33(4), 741-753.

Chaudhuri, A., Routray, A., \& Kar, S. (2012, December). Effect of sleep deprivation on estimated distributed sources for Scalp EEG signals: A case study on human drivers. In 2012 4th International Conference on Intelligent Human Computer Interaction (IHCI) (pp. 1-6). IEEE.

Choudhury, S. R., Venkataramanan, S., Nemade, H. B., \& Sahambi, J. S. (2005). Design and development of a novel EOG biopotential amplifier. IJBEM, 7(1), 271-274.

Constable, P. A., Bach, M., Frishman, L. J., Jeffrey, B. G., \& Robson, A. G. (2017). ISCEV Standard for clinical electro-oculography (2017 update). Documenta Ophthalmologica, 134(1), 1-9.

Crescentini, M., Thei, F., Bennati, M., Saha, S., de Planque, M. R., Morgan, H., \& Tartagni, M. (2014). A distributed amplifier system for bilayer lipid membrane (BLM) arrays with noise and individual offset cancellation. IEEE transactions on biomedical circuits and systems, 9(3), 334-344.

Dasgupta, A., Chakraborty, S., \& Routray, A. (2017). A two-stage framework for denoising electrooculography signals. Biomedical Signal Processing and Control, 31, 231-237.

Kim, Y., Doh, N. L., Youm, Y., \& Chung, W. K. (2007). Robust discrimination method of the electrooculogram signals for human-computer interaction controlling mobile robot. Intelligent Automation \& Soft Computing, 13(3), 319-336.

Lathi, B. P. (2006). Sinais e sistemas lineares-2. Bookman.

Lee, H. D. (2005). Seleção de atributos importantes para a extração de conhecimento de bases de dados (Doctoral dissertation, Universidade de São Paulo).

Mala, S., \& Latha, K. (2013). Efficient classification of eog using cbfs feature selection algorithm. ERCICA'13, 800-806.

Miotec Equipamentos Biomédicos (2010). Miotool 200/400 Manuais do Usuário. Rev. D.

Nishida, E. N. (2017). Propriedade da Filtragem de Savitzky-Golay Aplicadas na Identificação de Complexos QRS em Sinais de Eletrocardiograma.

Pereira, A. S., Shitsuka, D. M., Parreira, F. J., \& Shitsuka, R. (2018). Metodologia da pesquisa científica. UFSM.

Sadeghi, M., \& Behnia, F. (2018). Optimum window length of Savitzky-Golay filters with arbitrary order. arXiv preprint arXiv:1808.10489.

Vogt, J. E. (2015). Unsupervised structure detection in biomedical data. IEEE/ACM transactions on computational biology and bioinformatics, 12(4), 753-760.

Zhao, A. X., Tang, X. J., Zhang, Z. H., \& Liu, J. H. (2014, June). The parameters optimization selection of Savitzky-Golay filter and its application in smoothing pretreatment for FTIR spectra. In 2014 9th IEEE Conference on Industrial Electronics and Applications (pp. 516-521). IEEE. 